Biology and complementarity in Niels Bobr's philosophy

\title{
Biología y filosofía de la complementariedad en Niels Bobr
}

\author{
Karim J. Gherab Martín \\ Universidad Rey Juan Carlos \\ karim.martin@urjc.es
}

DOI: https://doi.org/10.15366/bp.2020.24.023

Bajo Palabra. II Época. № 24. Pgs: 449-474

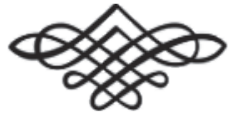


Recibido: 20/04/2019

Aprobado: 12/08/2020

\section{Resumen}

El presente artículo analiza la filosofía de la complementariedad de Niels Bohr en el campo de la biología. Para ello, muestra como surge el concepto de complementariedad en la física cuántica y cómo Bohr lo generaliza para aplicarlo a la biología. Asimismo, el artículo busca las fuentes filosóficas que dan origen a la filosofía de la complementariedad y concluye que dicha filosofía se orienta hacia el análisis epistemológico, y no el ontológico, de los objetos subatómicos y de los seres vivos.

Palabras clave: Niels Bohr, filosofía de la complementariedad, biología, teleología, fisica cuántica, vitalismo.

\section{Abstract}

This article discusses Niels Bohr's philosophy of complementarity in the field of biology. To do this, it shows how the concept of complementarity arises in quantum physics and how Bohr generalizes it so that to apply it to biology. Likewise, the article looks for the philosophical sources that give rise to the philosophy of complementarity and concludes that said philosophy is oriented towards an epistemological, rather than an ontological, analysis of subatomic objects and living beings.

Keywords: Niels Bohr, philosophy of complementarity, biology, teleology, quantum physics, vitalism. 


\section{Introducción}

EN UNA CONFERENCia pronunciada en Como, Italia, en 1927, titulada "The Quantum Postulate and the Recent Development of Atomic Theory" ", el premio Nobel de Física Niels Bohr introdujo por primera vez en público ${ }^{2}$ el concepto de complementariedad. La idea de la complementariedad se refiere sobre todo a la teoría cuántica que, en los últimos tres años (1925-1927), se había convertido ya en mecánica cuántica.

El concepto de complementariedad de Bohr fue la primera interpretación filosófica de la mecánica cuántica y está en la base de lo que posteriormente se llamó "interpretación de Copenhague", que fue apoyada por otros muchos protagonistas de la revolución cuántica: Heisenberg, Pauli, Born y Dirac, por mencionar sólo unos pocos. Bohr, sin embargo, no consideró su punto de vista como una interpretación, sino más bien como una explicación detallada de las posibilidades de descripción y observación dentro de la mecánica cuántica. El concepto de complementariedad establecía las condiciones de observación de los objetos del tamaño de un átomo y, por consiguiente, debe ser considerado como una teoría epistemológica. De hecho, el propio Bohr declaraba que la física cuántica nos había dado una "lección epistemológica" (Bohr, 1955a; 1955b) y que dicha lección podía extrapolarse a otros campos más allá de la física, en concreto a la biología, a la psicología y a la antropología. Aquí trataremos el caso de la biología.

Bohr se interesó por la filosofía y por la biología desde muy joven, mucho antes de convertirse en un referente mundial en la física. Ya desde pequeño, su padre Christian Bohr, a la sazón catedrático de fisiología en la Universidad de Copenhague, permitía a Niels y a su hermano Harald asistir a las tertulias que organizaba en casa con eminentes intelectuales daneses (Favrholdt, 1999: XXVIII), tales como Harald Høffding, Vilhem Thomsen, Johan Henrik Chievitz y Carl Lange ${ }^{3}$. En estas reuniones eran frecuentes los debates sobre biología y filosofía, y en concreto sobre la controversia entre mecanicismo y vitalismo heredada del siglo XIX (Lenoir, 1982).

\footnotetext{
${ }^{1}$ Conferencia celebrada el 16 de septiembre de 1927 en ocasión de los 100 años transcurridos desde el fallecimiento de Alessandro Volta. Fue publicada un año más tarde en Bohr (1928).

2 Bohr utiliza el término 'complementariedad' por primera vez (Pais, 1991: 311) en una carta de Bohr a Wolfgang Pauli, con fecha 13 de agosto de 1927.

3 Johan Henrik Chievitz y Carl Lange eran ambos profesores de anatomía.
} 
El presente artículo consta de tres partes: la primera analiza el concepto de complementariedad tal y como lo concibió Bohr para la física y trata sobre los filósofos que influyeron en Bohr (concretamente, Kant, Kierkegaard, James y Høffding); la segunda presenta las actividades relacionadas con la biología que llevó a cabo a lo largo de su vida y describe la filosofía de la biología de Bohr, que no es otra cosa que la aplicación de la noción de complementariedad (previamente aplicada a la física cuántica) en la biología; la tercera presenta unas conclusiones a modo de síntesis de las dos primeras. La conclusión es que la filosofía de la biología de Bohr no se enmarca en el plano ontológico, sino en el epistemológico. Así, veremos que Bohr podría ser adscrito al reduccionismo ontológico, pero en ningún caso ser considerado como reduccionista epistemológico.

\section{La filosofía de la complementariedad de Niels Bohr.}

I927 ES CONSIDERADo como el año en el que Niels Bohr y el físico alemán Werner Heisenberg culminaron un esfuerzo sin parangón en la historia de la ciencia. Los secretos del átomo se desvelaron por fin, tras resistirse 27 años a muchas de las mentes más brillantes del mundo. En 1900, el físico alemán Max Planck postuló la existencia de cuantos de energía (que los físicos simbolizan con la letra $\hbar$ ) para poder explicar un fenómeno conocido como radiación del cuerpo negro, dando comienzo al cisma cuántico. Entre medias, científicos de la talla de Albert Einstein, Ernest Rutherford, Erwin Schrödinger o Max Born, entre otros, pavimentaron el camino que conduciría al éxito a la generación cuántica.

Para entender de donde parte el concepto de complementariedad propuesto por Bohr es necesario analizar primero la aportación de Heisenberg. El alemán presentó ese mismo año su famoso principio de indeterminación (también conocido como principio de incertidumbre), que corresponde a la ecuación siguiente:

$$
\Delta x . \Delta p \geq \frac{\hbar}{2}
$$

Esta expresión matemática es, junto con la ecuación de Schrödinger de 1926, el pilar fundamental sobre el que se asienta todo el edificio teórico de la física cuántica. Tanto Schrödinger como Heisenberg recibieron el premio Nobel por el descubrimiento de estas ecuaciones. Dejando de lado a Schrödinger y centrándonos en Heisenberg, la ecuación de arriba nos dice grosso modo lo siguiente: no podemos conocer la posición ( $\mathrm{x}$ ) y la velocidad ( $\mathrm{p}$ ) de una partícula subatómica 
(o sea, cuántica) con una precisión mayor que la cantidad $\hbar / 2$, donde $\hbar$ es la constante de Planck. Dicho de otro modo: la incertidumbre sobre la posición $(\Delta x)$ y sobre la velocidad $(\Delta p)$, medidas en el mismo instante, es siempre superior a $\hbar / 2$. En otras palabras, cuanto mayor sea la precisión de la medición de la variable $x$, mayor será la imprecisión de la medición de la variable $p$, y viceversa. Siempre con el límite $\hbar / 2$ impidiendo la infinita precisión en la medición conjunta de ambas variables.

Es importante señalar que esta ecuación no representa un límite práctico subsanable con la mejora de las técnicas experimentales o con el desarrollo de nuevos instrumentos científicos. Muy al contrario, hoy sabemos que se trata de un límite que nos impone la propia Naturaleza. Pero esto en 1927 no estaba aún del todo claro. Esta falta de claridad en cómo interpretar la ecuación de Heisenberg condujo a los célebres debates filosóficos que enfrentaron a los partidarios de Einstein y a los partidarios de Bohr a lo largo de casi todo el siglo XX. Para Einstein y sus seguidores (llamados realistas) la imprecisión se reducía a un problema técnico. Para Bohr y sus seguidores (la llamada escuela de Copenhague) el problema no era técnico, sino consustancial al mundo físico ${ }^{4}$. La interpretación de Bohr es la que hace uso del concepto de complementariedad para explicar la ecuación de Heisenberg. Dicho de otro modo, Bohr intentó expresar en lenguaje filosófico lo que Heisenberg expresó en forma matemática.

El hecho de que no se puedan medir ambas variables con precisión infinita, y que esta limitación sea ontológica, tiene una consecuencia importante: el movimiento de los objetos subatómicos carece de trayectoria en el marco espaciotemporal, lo cual nos obliga a renunciar al principio de causalidad en sentido clásico. En el lenguaje de los positivistas lógicos, diríamos que carece de significado hablar de objetos cuánticos moviéndose en el espacio y en el tiempo. Bohr lo expresó del modo siguiente:

- Ruptura con el principio de causalidad: cualquier intento de observar un objeto cuántico viene acompañado por una "ruptura total con la descripción causal de su comportamiento dinámico” (Bohr, 1928).

- Ruptura con la noción de realidad objetiva: "una realidad independiente en el sentido físico ordinario no puede atribuirse a los fenómenos ni a las agencias de observación [agencies of observation]" (Bohr, 1928).

\footnotetext{
4 "No se trata aquí de una limitación práctica en la precisión de las mediciones, sino de un aspecto de las leyes de la naturaleza, asociado con el cuanto de acción, que establece un límite inferior para la interacción entre los objetos y los instrumentos de medición" (Bohr, 1952).
} 
Inicialmente, Bohr y Heisenberg recurrieron a una imagen un tanto simplista, de la que posteriormente intentaron alejarse con argumentos filosóficos más sofisticados. Dicha imagen consistió en recurrir a una metáfora teatral:

el propio paralelismo psicofísico, tal como fue considerado por Leibniz y Spinoza, ha visto ampliar su marco como consecuencia del desarrollo de la física atómica, que nos ha forzado, frente al problema de la explicación, a tomar una actitud que recuerda el antiguo apotegma de que cuando se busca la armonía en la vida no puede olvidarse que somos a la vez espectadores y actores en el gran drama de la existencia. (Bohr, 1964: 77)

La física clásica (newtoniana) postulaba una nítida separación entre el sujeto que observa y los objetos observados. Así, el científico sería un puro espectador que no influye en su entorno; sin embargo, la ecuación de Heisenberg parecía sugerir la inevitabilidad de la perturbación del objeto observado. Bohr (1964: 49) advertía de "la imposibilidad de cualquier separación precisa entre el comportamiento de los objetos atómicos y su interacción con los instrumentos de medida que sirven para definir las condiciones bajo las cuales se manifiesta el fenómeno".

Probablemente inspirado en la filosofía kantiana, el físico danés estaba pues, ya en 1929, redefiniendo el concepto de fenómeno físico dejando la cuestión como sigue:

La magnitud finita del cuanto de acción impide hacer una distinción neta entre el fenómeno y el instrumento de observación, distinción que subyace al concepto ordinario de observación y que por lo tanto está en la base de las ideas clásicas del movimiento. (Bohr, 1988: 60-61)

Como es bien sabido por físicos y filósofos de la física, los montajes experimentales para detectar objetos cuánticos condicionan el fenómeno observado: un tipo de experimentos revelan su condición corpuscular; mientras que otro tipo de experimentos revelan su condición ondulatoria. Es lo que se conoce como dualidad onda-corpuisculo. Puesto que el fenómeno observado (una partícula o una onda) depende del montaje experimental o instrumento utilizado, era obvio especular que el instrumento debía formar parte del fenómeno y que, por tanto, podía consignarse como parte del objeto estudiado. Gerard Holton (1982: 123) afirma que Bohr se dio perfecta cuenta de que "tan pronto como [el observador] dispone sus herramientas de observación en la mesa de trabajo, el sistema que ha elegido para someter a observación y los instrumentos de medida que le sirven para hacer el trabajo ya forman un todo inseparable".

Hemos visto hasta aquí que el principio de indeterminación (o incertidumbre) de Heisenberg nos señala un límite en la precisión que podemos tener del valor ob- 
jetivo de las cantidades $\mathrm{x}$ y $\mathrm{p}$. Es decir, la precisión de las medidas sobre $\mathrm{x}$ y sobre $\mathrm{p}$ son mutuamente excluyentes; pero tanto $\mathrm{x}$ como $\mathrm{p}$ son necesarias en la descripción clásica de los fenómenos. Asimismo, hemos visto que los fenómenos cuánticos observados son dependientes del tipo de montaje experimental: o vemos la manifestación ondulatoria de los objetos subatómicos, o vemos su manifestación corpuscular; pero jamás se manifiestan ambas formas a la vez. Por consiguiente, podemos afirmar también que la imagen corpuscular y la imagen ondulatoria son mutuamente excluyentes. Sin embargo, ambas son necesarias para obtener una caracterización completa del sistema cuántico bajo observación. Estamos pues en disposición de dar una definición de la filosofía de la complementariedad de Bohr: dos descripciones son complementarias si y solo si requieren de arreglos experimentales mutuamente excluyentes, pero son necesarias ambas para una comprensión completa de los fenómenos.

Para describir el problema en cuestión, a Bohr (1928: 590; 1933: 31) le gustaba trazar un paralelismo entre la situación cuántica y la teoría especial de la relatividad de Einstein, señalando que la dependencia de los fenómenos cuánticos con los instrumentos de medida es similar a lo que sucede con las contracciones y dilataciones espaciotemporales relativas a cada marco de referencia en la teoría de Einstein. Unos años después, en 1933, su discípulo austriaco Wolgang Pauli ${ }^{5}$ señalaría en favor de Bohr que "en analogía a la expresión teoría de la relatividad, uno podría llamar a la moderna teoría cuántica teoría de la complementariedad" (Pauli, 1933: 89).

Una vez analizado y definido el concepto de complementariedad en el ámbito de la física, podemos preguntarnos cual pudo ser el origen de esta idea. Ya hemos visto que Christian Bohr, padre de Niels, era catedrático de fisiología en la Universidad de Copenhague y organizaba asiduamente reuniones en casa para el club informal al que pertenecía. Entre los invitados solía estar Harald Høffding, profesor de filosofía de la misma universidad y amigo íntimo de la familia, y aunque Niels Bohr era aún joven, tenía el permiso de su padre para presenciar dichas reuniones y, por consiguiente, escuchar lo que allí se dijera. Con el tiempo, la admiración de Niels por Høffding fue creciendo hasta el punto de que decidió asistir a las clases de filosofía que éste impartía en la universidad, por lo que no debe resultar extraño que las ideas de Høffding influyeran sobre sus pensamientos de juventud.

Høffding tenía interés en la psicología y mantuvo contactos con el psicólogo y filósofo William James. De hecho, como resultado de un viaje a Estados Unidos para visitar a James en 1904, éste escribió el prefacio de la traducción inglesa del libro Problems of Philosophy de Høffding en 1905. Høffding tenía en alta estima a

\footnotetext{
5 Premio Nobel de Física en 1945 por el descubrimiento del principio de exclusión, característico de un tipo de partículas cuánticas y necesario para comprender la disposición de los electrones en los átomos. Actualmente, se le conoce como principio de exclusión de Pauli.
} 
James y es muy posible que la influencia posterior de los trabajos de James sobre Bohr se concretara a través de Høffding. Es muy probable que el gusto de Bohr por la "dialéctica cualitativa" de Kierkegaard, basada "en la declaración de que las contradicciones en la vida y en la naturaleza son rígidas e imposibles de superar" (Selleri, 1986: 101), fuera heredado también de Høffding puesto que éste era considerado en Dinamarca como uno de los comentaristas eminentes de Kierkegaard, también danés y nacido en Copenhague. Además, su segunda obra importante publicada fue el libro Kierkegaard als philosoph, en 1892. De hecho, en uno de sus libros, History of Modern Philosophy ${ }^{6}$, Høffding escribía un párrafo que refleja el paralelismo entre lo que él interpretaba de Kierkegaard en lo referente al problema de la discontinuidad y lo que Bohr se encontraría años más tarde ${ }^{7}$ al enfrentarse con los saltos cuánticos ${ }^{8}$ :

No tiene lugar ningún desarrollo gradual dentro de la esfera espiritual como el que podría explicar la transición desde la deliberación a la decisión [...]. La elección en sí llega con una sacudida, con un salto, en el que se propone algo completamente nuevo (una nueva cualidad). Solamente hay continuidad en el mundo de las posibilidades; en el mundo de la realidad la decisión siempre llega a través de una rotura en la continuidad. [...] Casi parecería seguirse de esto que el salto en sí no puede ser observado. (Høffding, citado en Holton, 1982: 154)

Se observa un gran parecido entre lo expuesto por Høffding y la extrańa mecánica del mundo cuántico: la deliberación suele acomodarse a un número finito de alternativas que entrañan una decisión; cada decisión corresponde a una elección de entre las alternativas estudiadas; la "continuidad en el mundo de las posibilidades" describe una superposición de las alternativas a considerar; se produce una rotura de la continuidad en el instante de realizar la elección, un salto que no puede ser observado porque no hay una relación causal clara y evidente. Para un físico cuántico no es difícil ver en estas palabras lo siguiente: la deliberación es la superposición de los estados definidos que representan las distintas alternativas y salta a una elección (el valor medido) en el instante de la decisión (el acto de medir), adquiriendo nuevas cualidades (del aspecto ondulatorio continuo al aspecto corpuscular discreto) en una transición discontinua que no puede observarse y cuya causalidad no es explícita.

\footnotetext{
${ }^{6}$ Libro editado y traducido al inglés por B. E. Meyer.

7 El libro de Høffding aparece en 1893 y Bohr propone el principio de complementariedad en 1927.

${ }^{8}$ Niels Bohr recibió precisamente el premio Nobel en 1922 por su propuesta del modelo atómico de estados estacionarios con órbitas cuantizadas. Su trabajo, publicado en Bohr (1913a; 1913b; 1913c), presentaba un modelo estable para los átomos, postulando la existencia de órbitas con energías cuantizadas en múltiplos de $\hbar$. Según el modelo atómico de Bohr, el paso de un electrón de una órbita a otra se producía mediante saltos (los llamados saltos cuánticos), y no mediante trayectorias continuas en el espacio-tiempo. En otras palabras, el salto del electrón se produce mediante una ruptura de la continuidad espaciotemporal.
} 


\section{Añadía Høffding en el mismo libro:}

Kierkegaard llegó a considerar cada vez más como criterio de la sublimidad y valor de una concepción de la vida la capacidad de admitir en su seno grandes contrastes y de tolerar el sufrimiento que esto conlleva. (Høffding, citado en Holton, 1982: 153)

Sin llevar el significado de esta cita demasiado lejos, es interesante observar, como sugieren Holton (1982) y Selleri (1986: 101), el parecido existente entre la capacidad de admitir en el seno de la vida grandes contrastes, como proponía Kierkegaard, y el atrevimiento necesario de Bohr para admitir en la física como complementarias dos concepciones aparentemente irreconciliables (ondas vs corpúsculos) desde la época de Christiaan Huygens e Isaac Newton.

Las influencias que Bohr recibió de los escritos de William James son mucho más evidentes y es muy posible que el mismo término 'complementariedad' provenga del relato que hace el autor norteamericano en su libro The Principles of Psychology, publicado en 1890, de un experimento psicológico. El experimento trataba de mostrar la existencia de una conciencia secundaria totalmente separada de la conciencia normal, pero visible solo bajo ciertas condiciones, si se anestesiaba al paciente mediante anestesia histérica, es decir, inhibiendo la percepción natural de la vista, el oído, el tacto, etc. Para comunicarse con la conciencia secundaria o subconsciente del paciente, el psicólogo francés Pierre Janet se ponía detrás de él y le susurraba al oído las tareas a realizar, mientras se mantenía ocupada la consciencia normal del paciente con una conversación en voz alta con otra persona que tenía enfrente. El relato de William James es el siguiente:

M. Janet ha demostrado esto de forma elegantísima en Lucie, uno de sus sujetos. [...] Durante el trance cubrió su regazo con cartas, cada una de las cuales llevaba un número. Le dijo entonces que al despertarse no vería ninguna carta cuyo número fuese múltiplo de tres. Esta es la que se llama normalmente "sugestión post-hipnótica", bien conocida actualmente, y para la que Lucie constituía un sujeto bien adaptado. De acuerdo con esto, cuando fue despertada y se le preguntó sobre las cartas que tenía en el regazo, las contó y dijo que veía solamente aquellas cuyo número no era múltiplo de tres. Era ciega al 12, 18, 9, etc. Pero cuando el yo subconsciente fue interrogado siguiendo el método usual de ocupar al yo superior con otra conversación, la mano escribió que las únicas cartas en el regazo de Lucie eran las numeradas $12,18,9$, etc., y al pedírsele que cogiese todas las cartas que había allí, recogió estas y dejó las otras. Análogamente, cuando se le sugería a la Lucie subconsciente la visión de ciertas cosas, la Lucie normal se quedaba de repente total o parcialmente ciega. (James, citado en Holton, 1982: 147-148)

Como conclusión del experimento de las dos conciencias recién descrito, James introdujo el término 'complementariedad' en la psicología: 
Debe admitirse, por tanto, que, al menos en ciertas personas, la posible conciencia total puede escindirse en dos partes que coexisten pero que se ignoran mutuamente y comparten los objetos del conocimiento entre sí. Más notable todavía es que son complementarias. Demos un objeto a una de las conciencias y eso mismo hace que lo retiremos de la otra $\mathrm{u}$ otras. Con excepción de una reserva común de información, como el dominio del lenguaje, etc., aquello que conoce el yo superior lo ignora el yo inferior, y viceversa. (Citado en Holton, 1982: 148)

Parece bastante sugestiva la idea de tomar, como proponen Holton (1982: 147), Meyer-Abich (1965) y Jammer (1966), este párrafo como el origen del término 'complementariedad' que Bohr usaría posteriormente para resolver la paradoja cuántica, si bien no están de acuerdo con esta hipótesis ni Rosenfeld (1967), ni Pais (1991: 24, 310-311). Que Bohr leyó a James y que sintió gran admiración por él parece estar fuera de toda duda (Pais, 1991: 424), pero Heisenberg, en una entrevista (Holton, 1988) realizada por T. S. Kuhn el 11 de febrero de 1963, tiene sus dudas sobre si Bohr leyó el capítulo "El flujo del pensamiento" del libro de James The Principles of Psychology antes o después de concebir el vocablo 'complementariedad'. Como hemos dicho, Pais (1991: 424) discrepa firmemente acerca de las posibles influencias de filósofos sobre Bohr ya que, afirma, Bohr "nunca se preocupó mucho ni estaba al corriente de lo que los filósofos profesionales tuvieran que decir" (Pais, 1991: 24). Pero no es este experimento el único relato de James que muestra cierta afinidad con las ideas de Bohr, sino que su filosofía también permite sacar algunas conclusiones adicionales. James analizó la manera en que discurre el pensamiento y se dio cuenta que este fluye de forma continua puesto que en el momento en que se intenta congelar el pensamiento y atomizarlo para captar la esencia de su significado se pierde la continuidad que le da su todo sentido. Seccionar el pensamiento equivale pues a aniquilarlo:

Dejemos que alguien intente seccionar un pensamiento por la mitad y echar un vistazo al corte y verá lo difícil que es la observación introspectiva de la región transitiva... [...] Intentar un análisis introspectivo en estos casos es de hecho como... tratar de encender la luz rápidamente para ver cómo es la oscuridad. (James, citado en Holton, 1982: 146)

Esta idea de James se asemeja bastante a una de las aplicaciones hechas por Bohr del principio de complementariedad en el campo de la biología (Heisenberg, 1971: 126), años más tarde de hacer lo propio en la física. Es momento pues de analizar cómo Bohr proyectó su principio de complementariedad a la biología e intentó convertir sus ideas en un sistema filosófico aplicable a cualquier aspecto de la vida. 


\section{La filosofía de la complementariedad aplicada a la biología}

DURANTE LOS ÚlTIMOS TREINTA AŃOS DE SU VIDA, y gracias a su enorme prestigio mundial en calidad de Nobel y a sus excelentes relaciones con la Fundación Rockefeller (Pais, 1991: 255-260), Bohr aprovechó muchas oportunidades para difundir sus ideas sobre complementariedad a otros terrenos allende la física ${ }^{9}$, extrapolando sus argumentos acerca de la inseparabilidad entre el objeto atómico y el instrumento de medida del científico al caso más general de la distinción filosófica entre objeto y sujeto:

Espero, no obstante, que la idea de complementariedad sea adecuada para caracterizar la situación, que conlleva una analogía profunda con la dificultad general en la formación de ideas humanas, inherente a la distinción entre sujeto y objeto. (Bohr, 1928: 590)

La primera exposición exhaustiva de Bohr sobre biología fue su conferencia " $\mathrm{Li}$ ght and Life", presentada en agosto de 1932, en el II Congreso Internacional de Fototerapia en Copenhague ${ }^{10}$. El físico belga Léon Rosenfeld, discípulo y amigo personal de Bohr, ha escrito lo siguiente ${ }^{11}$ en relación con esta conferencia:

Esta [conferencia] tenía un atractivo especial para él: había quedado profundamente impresionado por las opiniones de su padre sobre el tema, y estaba visiblemente feliz de poder ahora abordarlas y darles una formulación más adecuada. Su padre [Christian Bohr], en reacción contra el materialismo mecanicista de principios de siglo, habia defendido enérgicamente el punto de vista teleológico en el estudio de la fisiologia: argumentó que, sin el conocimiento previo de la función de un órgano, no hay esperanza de desentrañar su estructura para los procesos fisiológicos de los cuales es la semilla. Al mismo tiempo, subrayó, con toda la autoridad de una vida dedicada al análisis de los aspectos físicos y químicos de tales procesos [fisiológicos], que era igualmente imperiosa la necesidad de llevar este análisis [físico y químico] al límite con los medios técnicos de investigación que estén a nuestro alcance...

Estas reflexiones llegaron tan cerca como cabría esperar en el momento de establecer una relación de complementariedad entre el lado fisico-químico de los procesos vitales, gobernado por el tipo de causalidad que [los científicos] están acostumbrados a anunciar como el verdaderamente científico, y el aspecto propiamente funcional de estos procesos, dominado por una causalidad teleológica o finalista. En el pasado, los dos puntos de vista, bajo diversas formas, siempre se habían puesto en fuerte oposición entre sí, la opinión general era que

\footnotetext{
9 "Bohr dedicó una cantidad considerable de intenso trabajo a explorar las posibilidades de aplicación de la complementariedad a otros campos del conocimiento; a esta tarea no le concedía menos importancia que a sus investigaciones puramente físicas y tampoco era menor la satisfacción que obtenía con sus logros en este terreno" (Rosenfeld, citado en Holton, 1982: 158).

${ }^{10}$ El texto de la conferencia fue publicado un año después, en Bohr (1933).

11 Todas las cursivas son mías, para enfatizar las expresiones que son clave para entender la aplicación del concepto de complementariedad en la filosofía de la biología de Niels Bohr.
} 
uno de ellos debia prevalecer por exclusión del otro, que no habia lugar para ambos en la ciencia de la vida. Niels Bohr podía ahora seńalar que esta última creencia era solo el resultado de una concepción de la lógica que los físicos habían revelado como excesivamente rigurosa, y que el marco más amplio de complementariedad parecía particularmente adecuado para acomodar los dos puntos de vista, y además hacerlo posible sin contradicción aprovechándose de ambos, muy en la línea del espíritu de las ideas de su padre. (Rosenfeld, 1967: 132-133)

Por otro lado, unos de sus principales biógrafos, el danés Jan Faye, señala que lo que dijo Niels Bohr en esa conferencia "sobre biología sonaba como el eco de Høffding” (Faye, 1991: 158). Høffding, al igual que Christian Bohr, afirmaba que la vida no es reducible a las leyes de la física y la química porque lo característico de los organismos vivos es que son totalidades indivisibles, poseen su propia "individualidad" intrínseca; y la relación entre la totalidad y la suma de sus partes no puede explicarse en términos causales. Adoptando una posición muy próxima a la de Kant ${ }^{12}$, Høffding pensaba que la comprensión del fenómeno de la vida está por encima del umbral de la inteligibilidad humana. Pero Høffding tampoco pensaba que la comprensión profunda de las estructuras vitales y de las funciones de los organismos biológicos fuera alcanzable por vías distintas a las fisicoquímicas. En otras palabras, tampoco el vitalismo ${ }^{13}$ tendría cabida para Høffding. La vida no sería explicable ni por la vía del mecanicismo, ni por la vía del vitalismo.

Niels Bohr retomó la cuestión donde la dejaron su padre y Høffding, pero lo interesante del caso es que encontró en los dilemas filosóficos de la física cuántica nuevos y poderosos argumentos sobre los que apoyarse y robustecer la tesis filosófica de sus maestros. Volviendo a su discurso en el II Congreso Internacional de Fototerapia celebrado en Copenhague en 1932, conviene que rescatemos el extracto en el que compara el caso de la física con el de la biología y traza una similitud entre la irreducibilidad del cuanto de acción $\hbar$ en física cuántica y la irreducibilidad de la vida en biología ${ }^{14}$ :

En cada experimento con organismos vivos, debe permanecer una incertidumbre con respecto a las condiciones físicas a las que están sujetos, y esta idea sugiere que la libertad

\footnotetext{
12 McKaughan (2005: 513) apunta que Christian Bohr y Harald Høffding apoyaban el característico a priori kantiano de los conceptos teleológicos. Para otros trabajos que destacan la influencia de la filosofía de la biología de Kant sobre Christian Bohr y Harald Høffding, ver Faye (1991) y Roll-Hansen (2000). En particular, Roll-Hansen señala que la obra de Kant Crítica del juicio teleológico apuntaba ya al dilema entre mecanicismo y teleología: por un lado, la naturaleza de la comprensión humana exige que todo conocimiento estrictamente científico sobre la naturaleza debe ser mecanicista (es decir, apelar a causas eficientes); y por otro lado, lo característico de los organismos vivos es que tienen propósitos, y éstos sólo pueden explicarse teleológicamente. Por tanto, la filosofía de la biología kantiana contenía dos tipos de explicación incompatibles, dos métodos en conflicto: el mecanicismo y la teleología.

13 El vitalismo es una tesis ontológica que sostiene que existe alguna entidad o fuerza no-física que actúa dentro de los organismos vivos. Y sobre teorías animistas en la antigüedad, ver Hernández Artigas (2020).

14 Las cursivas son mías.
} 
mínima que debemos permitir al organismo a este respecto es lo suficientemente grande como para permitirle, digamos, ocultar sus secretos más profundos de nosotros. Desde este punto de vista, la existencia de la vida debe considerarse como un hecho elemental que no puede explicarse, sino que debe tomarse como un punto de partida en biología, de manera similar al cuanto de acción, que aparece como un elemento irracional desde el punto de vista de la física clásica [...]. La afirmación de la imposibilidad de una explicación física o quimica de la función que caracteriza a la vida sería, en este sentido, análoga a la insuficiencia del análisis mecánico para la comprensión de la estabilidad de los átomos. (Bohr, 1933: 458)

Bohr nos dice aquí que la vida es a la biología lo que $\hbar$ es a la física: en ambos casos se da una ruptura en la inteligibilidad del mundo real objetivo; ambas escapan a una explicación comprehensiva basada en las leyes causales propias de la visión mecanicista. La conclusión es que ni la visión mecanicista, ni la concepción vitalista en la biología son suficientes por separado, pero que, aún siendo excluyentes entre sí, son complementarias, alcanzando así Bohr una definición para la noción de complementariedad en la biología:

No estamos hablando ni del intento de trazar una analogía entre la vida y el simple funcionamiento de una máquina, ni de la idea de una fuerza vital mística, sino de dos aproximaciones que solo juntas agotan la posibilidad de incrementar nuestro conocimiento. En este sentido, las perspectivas mecanicista y vitalista pueden ser consideradas como complementarias. (Bohr, 1952: 971)

Además, Bohr buscaba similitudes entre el acto de medir en la física cuántica y el intento de diseccionar un ser vivo. En ambos casos, decía, se produce una intrusión en el objeto de estudio, y dicha intrusión o interacción destruye aquello que se pretendía observar, bien sea un átomo o un ser vivo. Tanto el átomo como la vida son entidades frágiles, susceptibles de ser perturbadas y desaparecer de golpe, en un salto análogo al cuántico. Es decir, si la física cuántica nos dice que los átomos no tienen trayectorias y continuidades espaciotemporales, la observación de los seres vivos nos enseña que el paso de la vida a la muerte se produce también en un salto, sin continuidad, sin retorno. Una vez el organismo "cruza" la desconocida y estrecha línea que separa la vida de la muerte, no es posible la vuelta atrás. En ambos casos se produce una ruptura, una discontinuidad, un salto.

Bohr señalaba que el mero intento de investigar a fondo y reducir el ser vivo a sus constituyentes físico-químicos básicos mataría al organismo:

Por lo tanto, si pudiéramos impulsar el análisis del mecanismo de los organismos vivos como en lo que respecta a los fenómenos atómicos, apenas deberíamos esperar encontrar características que difieran de las propiedades de la materia inorgánica. 
Con este dilema ante nosotros, debemos tener en cuenta, sin embargo, que las condiciones que se cumplen para la investigación en biología y la investigación en física no son directamente comparables, ya que la necesidad de mantener vivo el objeto de investigación impone una restricción en la primera [la investigación en biología], lo cual no tiene un equivalente en la segunda [la investigación en física]. Por lo tanto, no hay duda de que mataremos a un animal si tratamos de empujar la investigación de sus órganos hasta el punto de poder describir el papel desempeñado por cada átomo individual en las funciones vitales. (Bohr, 1933: 458)

Decía Bohr, además, que las analogías procedentes de la experiencia química ordinaria no proporcionan una explicación más satisfactoria de los organismos vivos que su comparación con artificios puramente mecánicos, como un aparato de relojería. Afirmaba que si fuéramos capaces de llevar el análisis del mecanismo de los organismos vivos tan lejos como el de los fenómenos atómicos, apenas podría existir la esperanza de encontrar un comportamiento distinto al de la materia inorgánica. Es decir, que si se descompone un cuerpo viviente hasta analizar sus constituyentes atómicos, éste no contendrá nada cualitativamente diferente de lo que puede estudiarse hoy en día en la física del estado sólido o en la química inorgánica; encontraremos los mismos átomos que los catalogados en la tabla periódica con las mismas fuerzas de enlace recíproco, siguiendo las mismas reglas cuánticas que siguen los cuerpos que estudian la física y la química, y entonces perderemos de vista aquello que da la vida a dicha estructura físico-química.

Es interesante advertir que esta manera de pensar era bastante habitual entre muchos físicos en la década de 1930. Conviene recordar que el descubrimiento de la estructura helicoidal del ADN y, por consiguiente, el nacimiento de la biología molecular, no se produciría hasta 1953. Trataremos cómo afectó el descubrimiento del ADN a las ideas de Bohr en breve, pero de momento veamos qué decía sobre estos asuntos otro eminente físico-filósofo, más o menos por la misma época: el astrofísico británico Sir Arthur Eddington, cuyos libros, en especial The Nature of the Physical World, fueron superventas en Inglaterra, EE.UU. y varios países europeos. Eddington especulaba con la existencia de "materia consciente" en algún lugar del cerebro que no fuera "idéntica en todos los aspectos a la materia inorgánica, lo cual reduciría al cuerpo a ser un autómata actuando independientemente de la consciencia” (Eddington, 1939: 181). Para estudiar la estructura atómica de un organismo vivo es necesario descomponerlo en sus partes constituyentes, cada vez con mayor precisión, reducirlo a la mínima expresión para poder captar su funcionamiento mecánico, si lo hay, de modo que al escudriñar un cuerpo biológico, una célula o una neurona, en busca de la vida o de la consciencia, decía Eddington que "nuestro método de análisis físico nos conduce a disecar esta célula en átomos análogos a 
los átomos de toda región no-consciente del universo" (Eddington, 1945: 95) ${ }^{15} \mathrm{y}$, por tanto, a plantearnos "la tremenda cuestión de cómo esta colección de átomos ordinarios puede ser una máquina pensante" (Eddington, 1929: 259).

Volvamos a Bohr y a su énfasis en los procesos e instrumentos de medición, en concreto al problema más general de la interacción entre objeto y observador/experimentador. Para Bohr, se puede admirar la vida siempre que no se interaccione de manera trágica con ella debido a que cuando queremos seccionar sus partes (o sea, los componentes atómicos y moleculares del organismo vivo) para contemplar de qué está compuesto ese ser vivo, solo alcanzamos a contemplar su muerte. En repetidas ocasiones, incluso poco antes del descubrimiento de la estructura del ADN y de la explicación del mecanismo de replicación asociado, Bohr $(1952 ; 1954)^{16}$ siguió insistiendo en que el análisis de los fenómenos de la vida por medio de los conceptos físicos está sometido a otra limitación esencial, que tiene su origen en el hecho de que si se desea llevar la observación de un organismo tan lejos como sea posible desde el punto de vista de la teoría atómica, es preciso efectuar sobre él una intervención que le mata. Como ya se ha dicho, existe una cierta incertidumbre que conduce irremediablemente a la idea de complementariedad como ocurre en la física cuántica, una incertidumbre concebida, al estilo del cuanto de acción finito ћ, como una cantidad mínima de cierta acción o función vital finita capaz de hacer saltar al organismo del estado vivo al estado muerto de manera discontinua.

Hasta aquí hemos analizado la manera en que Bohr desarrolló y proyectó su idea de la complementariedad desde la física a la biología. Pero es de interés detenernos momentáneamente para saber más sobre los ambiciosos proyectos biológicos en los que se embarcó en los años 30 hasta el comienzo de la Segunda Guerra Mundial. Veremos que Bohr fue un actor determinante para el posterior desarrollo de la biología molecular (Stent, 1989) y, por consiguiente, para el descubrimiento de la estructura del ADN. Lo irónico del caso es que su conferencia "Light and Life" de 1932, que ya hemos mencionado antes y que fue publicada en Bohr (1933), fue determinante para que algunos físicos ${ }^{17}$ decidieran dejar la física y pasarse a la biología ${ }^{18}$, culminando en un descubrimiento (el del ADN) que arruinaría su argu-

${ }_{15}$ Esta cita de Eddington ha sido recuperada de la traducción de su libro al castellano (Eddington, 1945: 95).

${ }^{16}$ La publicación de Bohr (1952) corresponde a una conferencia impartida en el II Congreso Internacional de Poliomielitis, celebrado en Copenhague el 3 de septiembre de 1951. La publicación de Bohr (1954) corresponde a su discurso en la ceremonia de apertura del VII Congreso Internacional de Radiología, celebrado en Copenhague el 19 de julio de 1953.

17 Dos casos paradigmáticos fueron Max Delbrück y Georg Karl von Hevesy, ambos galardonados con el premio Nobel ańos más tarde. Delbrück obtuvo el de Medicina en 1969 y Hevesy el de Química en 1943.

18 "La sugerencia de Bohr de una complementariedad en biología, análoga a la física, ha sido el motivo principal para el interés en biología de al menos un físico y posiblemente haya desempeñado un papel similar para otros físicos que entran en el campo de la biología” (Delbrück, 1949). 
mento acerca de la utilidad de la noción de complementariedad en la biología. En efecto, el descubrimiento del mecanismo de replicación de los seres vivos erradicó todo apoyo a argumentos vitalistas en biología, dando inicio a una nueva disciplina: la biología molecular (Fox Keller, 1990).

Como ya hemos dicho, resulta irónico pensar que Bohr se alejó del vitalismo como consecuencia de un programa de investigación que él ayudó a crear, puesto que influyó decisivamente en las motivaciones biológicas Max Delbrück, a la sazón un brillante joven físico alemán interesado en la cuántica. Muchos historiados de la ciencia ven en Delbrück el catalizador de la carrera que conduciría al descubrimiento del ADN. Los primeros contactos profesionales de Bohr con la biología como director del Instituto de Física Teórica en Copenhague comenzaron con motivo de la puesta en marcha de un programa de emergencia ${ }^{19}$ por parte de la Fundación Rockefeller (Fox Keller, 1990; Pais, 1991: cap. 17). Las contribuciones de la mencionada Fundación en el desarrollo de la biología molecular fueron notables, por eso merece la pena recordar las causas de su creación. El programa nació con el objetivo de dar refugio a los investigadores perseguidos en Alemania después del ascenso de Hitler a Canciller. Muchos judíos, y algunos no judíos, perdieron sus puestos en las universidades alemanas y sus vidas corrían peligro debido al creciente poder del partido nazi, lo que motivó innumerables fugas de cerebros hacia países occidentales. La sensación de inseguridad se contagió a otros países como Hungría, Polonia y Austria al ejecutarse los planes de expansión del Tercer Reich.

Bohr, que ya había tenido en 1932 la oportunidad de recibir en Copenhague la visita de Warren Weaver (quien ese mismo año fue nombrado director de la división de Ciencias Naturales de la Fundación Rockefeller por Max Mason, presidente de dicho organismo), realizó un viaje a los Estados Unidos en 1933 invitado por la American Association for the Advancement of Science. Allí Bohr habló con Mason en relación con el programa de emergencia y, aunque dicho programa sólo contemplaba ayudas específicas para temas relacionados con la biología, tanto Bohr como Mason contemplaron posibles vías de colaboración entre el Instituto del primero y la Fundación dirigida por el segundo:

En aquella ocasión Bohr sugirió atraer a su instituto “jóvenes capaces y perfectamente entrenados en matemáticas, física, y química, quienes bajo [su] dirección prestarían atención a alguna fase cuantitativa de problemas biológicos importantes". (Weaver, citado en Pais, 1991: 394)

\footnotetext{
19 Bohr fue apoyado económicamente para la creación del Instituto y los laboratorios asociados (tanto de física como de biología) con financiaciones sumamente generosas, tanto de la Fundación Rockefeller como de la Fundación Carlsberg.
} 
Bohr tenía amigos judíos en situación comprometida en Alemania y la Fundación podía servir de ayuda para ofrecerles una estancia en Copenhague colaborando en las investigaciones llevadas a cabo en el Instituto. Así pues, consiguió la ayuda necesaria por parte de la Fundación Rockefeller con el compromiso de dirigir las investigaciones físicas que realizaran los becados y aplicar los conocimientos adquiridos al campo de la biología. No hay duda del enorme atractivo que Bohr encontró en esta nueva fuente de financiación, pero no menos cierto es el interés que siempre mostró Bohr por la biología, un interés motivado por las consideraciones filosóficas anteriormente tratadas con relación a la oposición entre mecanicismo y vitalismo, y su posible resolución mediante el concepto de complementariedad.

Uno de los afortunados con las ayudas de la Fundación norteamericana fue el ya mencionado Von Hevesy, un físico húngaro, quien posteriormente desarrollaría sus investigaciones en Copenhague en temas relacionados con procesos radioactivos en biología. Hevesy, al igual que Bohr, había tenido la oportunidad de trabajar junto a Rutherford, el descubridor del núcleo atómico, y, por consiguiente, tenía un conocimiento de primera mano para acometer experimentos relacionados con procesos radiactivos. De modo que Hevesy, aprovechando la financiación que Bohr lograba de la Fundación Rockefeller, se dedicó a desarrollar y usar marcadores radiactivos en tejidos biológicos en el Instituto de Bohr: desde el análisis de sustitución de átomos de fósforo-32 ( $\left.{ }^{32} \mathrm{P}\right)$ en los huesos y en los dientes hasta el estudio de dichos procesos en el hígado y en el cerebro. Las investigaciones de Hevesy tomaron tanta importancia que Bohr conseguía cuantiosas sumas de dinero para financiar los aparatos necesarios para los experimentos biológicos de Hevesy, quien logró el Nobel de Química por "el uso de isótopos $\left[{ }^{32} \mathrm{P}\right]$ como indicadores en el estudio de procesos químicos". Para hacernos una idea del peso que tomaron las investigaciones de Hevesy dentro del Instituto de Bohr basta con recordar el relato de la física alemana Hilde Levi (en una entrevista concedida en 1974) en referencia a la situación del Instituto durante los diez años que duró la estancia de Hevesy en Copenhague, que finalizó en 1944. Levi, que logró un contrato en el Instituto de Física Teórica de Bohr para trabajar con Hevesy, decía lo siguiente:

Teníamos ratas y teníamos gatos y teníamos toda clase de extrañas cosas que de ningún modo encajaban con el Instituto de Bohr.

[...] Ya antes de que [Hevesy] se fuera había una sensación tanto en Hevesy como en Bohr, y no tengo duda de que debieron hablar ya en aquel tiempo, de que era un poco absurdo y un tanto fuera de lugar, que Hevesy intentara mantener un grupo trabajando en cuestiones biológicas en un ambiente tan claramente poco biológico como el Instituto de Bohr. (Levi, citada en Pais, 1991: 393) 
Más o menos por la misma época, concretamente entre 1932 y 1934, Max Delbrück, que recientemente había obtenido su doctorado en física teórica investigando en temas relacionados con la mecánica cuántica bajo la dirección de Max Born, visitaba frecuentemente el instituto de Bohr. Su primera visita se produjo en 1931 y duró aproximadamente seis meses, tiempo suficiente para que interiorizara por entero las ideas de Bohr sobre la complementariedad (Pais, 1991: 442). El propio Delbrück expresa retrospectivamente de la siguiente manera la afortunada influencia que causó en él la figura de Bohr:

Soy quizá el único de sus asociados de aquellos días que tomó [a Bohr] tan en serio que determinó mi carrera, cambiándome a la biología para buscar si había algo de verdad desde este punto de vista [la complementariedad]. (Delbrück, 1963: 41)

Durante su estancia en Copenhague en 1931 y, sobre todo, a raíz de la conferencia "Light and Life" de Bohr (aquí mencionada ya repetidas veces), a Delbrück le intrigó sobremanera el principio de complementariedad aplicado a la biología (Stent, 1989; Fox Keller, 1990; Stent, 1998; Roll-Hansen, 2000; McKaughan, 2005). Desde entonces, no dejó de pensar en la idea de una fuerza desconocida que estuviera ejerciendo un impulso vital más allá de las leyes establecidas por la mecánica cuántica. Así pues, Delbrück había llegado de Berlín con el objetivo de colaborar con Bohr únicamente en temas relacionados con la física, pero las ideas filosóficas de Bohr calaron tan hondo en él que decidió redirigir su carrera científica hacia la biología, y su propósito a partir de entonces consistió en confirmar o refutar las afirmaciones del físico danés, a saber, comprobar si era verdad que no podía haber, en el nivel atómico, configuraciones atómicas o moleculares distintas en los seres vivos a las apreciadas en la materia inerte. Judson (1996: 32) asegura que Delbrück fue el primer físico teórico que se atrevió, de manera sistemática, a bajar el nivel de estudio de la biología al nivel atómico, haciendo por ejemplo conjeturas acerca del número de átomos que, dispuestos de forma regular, podían rellenar el volumen ocupado por un gen.

Delbrück abandonó Europa en 1937 para proseguir sus investigaciones en Estados Unidos bajo el paraguas de la Fundación Rockefeller, convirtiéndose en profesor de biología en Caltech en 1946 gracias al ofrecimiento de George Beadle, director del departamento de biología. En el periodo 1937-1940, Delbrück ya había trabajado en Caltech, a donde había llegado con el objetivo (Judson, 1996: 32) de aplicar sus conocimientos de física a problemas biológicos, junto a Emory Ellis, que fue quien le introdujo en el mundo de los bacteriofagos, hasta que en 1940 aceptó un puesto como profesor de física en la Universidad de Vanderbilt en Nashville, Tennesse. Obviamente, dada su formación como físico, Delbrück pensó que su 
aportación a la biología sería especialmente significativa aplicando sus conocimientos de física a los organismos vivos, de modo que eligió como elemento de estudio los fagos, especialmente a raíz del descubrimiento y de la descripción hecha por Felix d'Hérelle de la multiplicación de un fago en el seno de una célula bacteriana. ¿La multiplicación de un fago implicaba la multiplicación de los constituyentes atómicos? Esta era una pregunta con importantes consecuencias en física en caso de respuesta afirmativa y Delbrück no era ajeno a ello. No parecía pues haber dudas acerca de las muchas respuestas que podía aportar la física a la resolución de los problemas biológicos, al menos para Delbrück, que, junto con el italiano Salvador Luria, creó el "grupo de los fagos" tras una reunión entre ambos en el verano de 1941 en Cold Spring Harbor, Long Islands. Sus motivaciones eran convincentes: Delbrück consideraba al bacteriofago como el más elemental de los sistemas biológicos, algo así como el átomo de hidrógeno en física. El paso dado por Delbrück al analizar los sistemas biológicos en el nivel de componentes atómicos animó a otro físico teórico, Erwin Schrödinger, a escribir su libro ¿Qué es la vida? que influyó decisivamente en el salto dado por innumerables físicos a la biología y que condujo, en opinión de un notable número de historiadores de la ciencia, al descubrimiento de la estructura del ADN. Al menos dos de los tres galardonados con el Nobel de Medicina de 1962 por el descubrimiento de la estructura del ADN, Francis Crick y Maurice Wilkins, han admitido haber dejado la física por la biología influidos por la lectura de este libro.

A raíz del descubrimiento de la estructura del ADN en 1953 y del mecanismo de replicación Bohr pareció renunciar al vitalismo (Pais, 1991: 443), si bien insistió en la complementariedad sustituyendo el vitalismo por la teleología, quizá como un intento de preservar el programa de su padre Christian Bohr, "un adorador de Goethe" según Oskar Klein (citado en Rozental, 1967: 13), quien además recuerda una conversación con Bohr en la que éste le mencionó la idea de su padre de que la teleología puede ser un punto de vista a la par con el de la causalidad a la hora de describir el comportamiento de los seres vivos [...], idea que jugaría más tarde un papel esencial en el intento de Bohr para clarificar la relación entre las formas que tienen el biólogo y el físico de describir la naturaleza" (Klein, 1967: 76).

Dejando de lado definitivamente cualquier mención a vitalismos de cualquier tipo, Bohr recuperó la idea de causalidad teleológica, en la que el efecto explica a la causa, en contraposición a la causalidad mecanicista, que antepone en el plano temporal la causa al efecto. Muchos años después, Heisenberg intentaba recordar uno de sus innumerables diálogos con Bohr en Copenhague. El siguiente extracto corresponde, al parecer, a un viaje en yate entre Copenhague y Svendborg, en compañía del anatomista Chievitz y del físico-químico (y patrón del barco) Bjerrum: 
[Bohr:] Ambos modos de pensar se contradicen entre sí. Porque en el primer caso [la causalidad teleológica] presuponemos que el acontecimiento está determinado por el objetivo al que sirve, por el fin al cual está dirigido; en el segundo [la causalidad mecanicista] creemos que el hecho está fijado por el hecho inmediatamente precedente, la situación inmediatamente anterior. Que ambas aproximaciones den al azar el mismo resultado, parece, sin embargo, inverosímil. Sin embargo, ambas formas de pensar se complementan mutuamente, pues en realidad sabemos desde hace tiempo que las dos son correctas, precisamente porque hay vida. La cuestión que se plantea para la biología no es, por tanto, cuál de los dos puntos de vista es el más auténtico, sino tan sólo cómo ha logrado la naturaleza el acoplamiento de ambos.

[...] Se puede afirmar también que los dos puntos de vista de que hemos hablado se refieren a situaciones complementarias de observación. En principio, podríamos medir, probablemente, la posición de cada átomo en una célula. Pero es imposible pensar en una medición semejante sin matar al mismo tiempo la célula viva. Lo que en definitiva sabríamos se reduciría simplemente al ordenamiento de los átomos en la célula muerta y no en la célula viva.

[Heisenberg:] Esta delimitación de las leyes biológicas y de las leyes fisico-químicas por la complementariedad me parece evidente.

(Heisenberg, 1972: 138-140)

Como hemos dicho, Bohr reemplazó el vitalismo por la causalidad teleológica, pero persistió en su idea de complementariedad. No obstante, sus discursos seguían apoyándose en argumentos cercanos a los kantianos ${ }^{20}$ que hemos visto, puesto que puso a partir de entonces especial énfasis en la idea de propósito (donde el énfasis de la cursiva en el siguiente extracto es, en esta ocasión, del propio Bohr):

Una descripción de la función interna de un organismo y su reacción a estímulos externos requiere de la palabra propósito, que es ajena a la física y la química... las actitudes denominadas mecanicistas y finalistas no son puntos de vista contradictorios, sino que exhiben una relación complementaria.

La sacudida del descubrimiento del mecanismo de replicación genético provocó que Bohr modificara sus argumentos por tres vías: por un lado, generalizó la noción de complementariedad a ámbitos muy alejados de la ciencia, tales como la política; por otro lado, relacionó la complementariedad con la complejidad de los organismos vivos; y finalmente, en mi opinión las más relevante de las tres, cambió su objeto de estudio, poniendo progresivamente más énfasis en la psicología que

\footnotetext{
${ }^{20}$ Recordemos el argumento dado por Kant en Crítica del juicio teleológico, donde afirma que lo característico de los organismos vivos es que tienen propósitos.
} 
en la biología y apuntando a la consciencia, y no tanto a la vida (cuyo mecanismo de replicación ahora era ya conocido), como elemento irreducible al que asociar las nociones de propósito y complementariedad. Así, el texto (inacabado) de su último discurso antes de morir, "Light and Life Revisited" ${ }^{21}$, finaliza enfatizando (Bohr, 1962: 198) la importancia de la consciencia, de los pensamientos, de los sentimientos y del Yo.

\section{Conclusiones}

El PRESENTE arTículo ha mostrado cómo Niels Bohr, uno de los padres fundadores de la física cuántica, intentó construir un edificio filosófico basado en el concepto de complementariedad surgido de los dilemas y aparentes contradicciones presentes en el mundo de los objetos y fenómenos subatómicos. Hemos trazado sus antecedentes filosóficos y analizado las figuras que influyeron en sus ideas de juventud. Hemos visto cómo Kant, Kierkegaard, James, Høffding y su padre, Christian Bohr, han influido más o menos en sus argumentos. En especial, la visión heredada del siglo XIX de la existencia de una oposición, quizás irresoluble, entre vitalismo/ teleología y mecanicismo.

Es evidente que la filosofía de la complementariedad, a pesar de atraer el interés de muchos físicos, historiadores y filósofos de la física, no ha tenido repercusión fuera de este círculo de expertos. Tal vez su mayor logro haya sido su mágica fuerza para provocar el trasvase de varios físicos hacia la biología, una fuerza que seguramente tiene que ver también con el inmenso carisma de su autor, Niels Bohr. Como decíamos, a pesar de su escaso impacto como sistema filosófico, la filosofía de la complementariedad (actualmente conocida como Interpretación de Copenhague entre los físicos) no ha sido superada por ninguna otra interpretación de la mecánica cuántica. Hoy conviven innumerables interpretaciones, todas ellas igual de válidas, para explicar lo que sucede en el mundo subatómico. $\mathrm{Y}$ aunque es verdad que la Interpretación de Copenhague ya no es la favorita de los físicos y filósofos de la física actuales, tampoco puede ser descartada. Probablemente, el motivo por el que la interpretación de Bohr no tiene ya seguidores, es porque renuncia a cualquier visión e intelección ontológica de la realidad. Es antirrealista, kantiana, positivista.

Tal y como hemos visto, la filosofía de la complementariedad sostiene que, para obtener una comprensión completa de los sistemas vivos, son igualmente necesarios dos modos de descripción mutuamente excluyentes: las descripciones mecanicista

${ }^{21}$ El 21 de junio de 1962 en el Instituto de Genética de la Universidad de Colonia. 
y finalista, que se complementan entre sí. Bohr afirmaba que la biología necesita dos ámbitos de investigación distintos para poder progresar: uno para describir los mecanismos físicos de los que dependen los fenómenos a nivel subcelular; y otro relacionado con la escala de los organismos y cómo estos organismos interactúan en sistemas más grandes, donde el concepto de propósito es relevante. Actualmente, sigue habiendo una tensión entre los que están a favor de reducir la biología a la física, y los que no: un enfoque reduccionista centrado en moléculas individuales que interaccionan entre sí de manera mecánica; y otro enfoque más sistémico, más holista, para estudiar niveles superiores de organización, de elevada complejidad.

Hasta donde hemos podido analizar, Bohr ni abrazó, ni rechazó el reduccionismo ontológico en el sentido de afirmar o negar que todo lo que existe está compuesto por un mismo conjunto de elementos fundamentales. Sin embargo, sí se opuso radicalmente al reduccionismo epistemológico, puesto que este asume que las unidades epistémicas tales como los conceptos, las leyes y las teorías de un determinado nivel de organización pueden derivarse mediante la implementación de las reglas de reducción de las unidades epistémicas que se aplican a niveles inferiores y más fundamentales. Adviértase que esto era lo que él negaba, a saber, que los conceptos, leyes y teorías de la biología o de la psicología pudieran derivarse de la física y de la química. En todo caso, podrían derivarse parcialmente, ya que la filosofía de la complementariedad nos constrińe a aceptar que la física y la química nos ofrecen sólo una descripción parcial de la realidad biológica. Necesitamos siempre su descripción complementaria (excluyente) para alcanzar la caracterización completa. Ambas descripciones son necesariamente excluyentes, advertía Bohr, pero sólo juntas nos revelan la verdadera comprensión de la realidad.

Quizás convenga, para equilibrar, acabar con dos opiniones contrarias a la filosofía de la complementariedad. Por un lado, el matemático Stanislaw Ulam:

Me parece que, como guía filosófica, la complementariedad es esencialmente negativa. Sólo puede consolar. Si sirve en verdad para algo más que para dar consuelos filosóficos, es algo que me inquieta. (Ulam, 2002: 176)

O la del propio Einstein refiriéndose a la noción de complementariedad aplicada a los objetos cuánticos:

Creer esto es lógicamente posible sin contradicción, pero resulta tan opuesto a mi instinto científico que no puedo abandonar la búsqueda de una concepción más completa. (Einstein, citado en Bohr, 1964: 76) 


\section{REFERENCIAS Bibliográficas}

Arana, Juan (ed.) (2020). "Niels Bohr: el hombre que revolucionó la ciencia clásica”. En J. Arana (ed.) (2020). La cosmovisión de los grandes científicos del siglo $X X$. Tecnos, Madrid.

Bohr, Niels (1988): La teoría atómica y la descripción de la naturaleza. Edición de Miguel Ferrero. Alianza, Madrid.

- (1964). Física atómica y conocimiento humano. Aguilar, Madrid.

- (1963). "Light and Life Revisited". ICSU Review 5, pp. 194-199.

- (1959). "Quantum physics and biology". Symposium on Models in Biology, in Bristol, 1959. Publicado con el título "Physical Models and Living Organisms" en W.D. McElroy \& B. Glass (eds.) (1961). Light and Life. Baltimore: Johns Hopkins University Press, pp. 1-3.

- (1955a). "Science and the Unity of Knowledge". En L. Leary (ed.) (1955), The Unity of Knowledge. New York: Doubleday \& Co., pp. 47-62

- (1955b). "Physical Science and Man’s Position”. Ingeniøren 64, pp. 810-814.

- (1954). "Address at the Opening Ceremony". Acta Radiologica 116 (Suppl.), pp. 15-18.

- (1952). "Medical Research and Natural Philosophy". Acta Medica Scandinavica 142 (Suppl.), pp. 967-972.

- (1933). "Light and Life". Nature 131, pp. 421-423, 457-459.

- (1928). "The Quantum Postulate and the Recent Development of Atomic Theory”. Nature 121 (Suppl.), pp. 580-590.

- (1913a). "On the Constitution of Atoms and Molecules, Part I". Philosophical Magazine 26 (151), pp. 1-24, https://doi.org/10.1080/14786441308634955.

- (1913b). "On the Constitution of Atoms and Molecules, Part II Systems Containing Only a Single Nucleus”. Philosophical Magazine 26 (153), pp. 476502, https://doi.org/10.1080/14786441308634993.

- (1913c). "On the Constitution of Atoms and Molecules, Part III Systems containing several nuclei”. Philosophical Magazine 26 (155), pp. 857-875, https://doi.org/10.1080/14786441308635031. 
Delbrück, Max (1963). "Biophysics". Commemoration of the fiftieth anniversary of Niels Bohr's first Papers on atomic constitution, pp. 41-67 (Copenhague, 8-15 July 1963).

- (1949). "A Physicist Looks at Biology". Transactions of the Connecticut Academy of Arts and Sciences 38, pp. 173-190.

Eddington, Arthur S. (1945). Nuevos senderos de la ciencia. Montaner y Simón, Barcelona.

- (1939). The philosophy of physical science. New York, The Macmillan Company.

- (1935). New pathways in science. New York, The Macmillan Company.

- (1929). The Nature of the Physical World. London: Cambridge University Press.

Favrholdt, David, (ed.) (1999). Niels Bohr Collected Works. Volume 10. Complementarity Beyond Physics (1928-1962). Elsevier Science, Amsterdam.

Faye, Jan (1991). Niels Bohr: His Heritage and Legacy. An Anti-Realist View of Quantum Mechanics. Springer Science, Dordrecht.

Fox Keller, Evelyn (1990). Physics and the Emergence of Molecular Biology. A History of Cognitive and Political Synergy". Journal of the History of Biology 23 (3), pp. 389-409.

Heisenberg, Werner (1972). Diálogos sobre la física atómica. La Editorial Católica, Madrid.

Hernández Artigas, Aniol (2020). "El pensamiento platónico del alma en la concepción médico-filosófica de Galeno". MEDICA Review 8 (1), pp. 1-9, https:// doi.org/10.37467/gka-revmedica.v8.2208

Holton, Gerald (1988). "The Roots of Complementarity". Daedalus 117 (3), pp. 151-197.

- (1982). Ensayos sobre el pensamiento científico en la época de Einstein. Alianza, Madrid.

Jammer, Max (1966). The Conceptual Development of Quantum Mechanics. New York: McGraw-Hill.

Judson, Horace Freeland (1996). The Eighth Day of Creation: Makers of the Revolution in Biology. Cold Spring Harbor Laboratory Press, New York.

Klein, Oskar (1967). "Glimpses of Niels Bohr as Scientist and Thinker". En S. Rozental (ed.) (1967), Niels Bohr. His Life and Work as Seen by His Friends and Colleagues. North-Holland, Amsterdam, pp. 74-93. 
Lenoir, Timothy (1982). The Strategy of Life: Teleology and Mechanics in Nineteenth-Century German Biology. Chicago: University of Chicago Press.

McKaughan, Daniel J. (2005). "The Influence of Niels Bohr on Max Delbrück: Revisiting the Hopes Inspired by 'Light and Life"'. Isis 96 (4), pp. 507-529, https://doi.org/10.1086/498591.

Meyer-Abich, Adolf (1965). Korrespondenz, Individualität und Komplementarität: eine Studie zur Geistesgeschichte der Quantentheorie in den Beiträgen Niels Bohrs Wiesbaden: Franz Steiner Verlag.

- (1955). "The principle of complementarity in biology". Acta Biotheoretica 11, pp. 57-74.

Pais, Abraham (1991). Niels Bohr's Times: in Physics, Philosophy, and Polity. Oxford University Press, New York.

Pauli, Wolfgang (1933). Handbuch der Physik. Springer, Berlin.

Roll-Hansen, Nils (2000). "The application of complementarity to biology: From Niels Bohr to Max Delbrück". Historical Studies in the Physical and Biological Sciences 30 (2), pp. 417-442, https://doi.org/10.2307/27757838.

Rosenfeld, Léon (1967). "Niels Bohr in the Thirties". En S. Rozental (ed.) (1967), Niels Bohr. His Life and Work as Seen by His Friends and Colleagues. North-Holland, Amsterdam, pp. 114-136.

- (1963): "Niels Bohr's contribution to epistemology". Physics Today 16 (10), pp. 47-54.

Rozental, Stefan (ed.) (1967). Niels Bohr. His Life and Work as Seen by His Friends and Colleagues. North-Holland, Amsterdam.

Selleri, Franco (1986). El debate de la teoría cuántica. Alianza, Madrid.

Stent, Gunther S. (1998). "Looking for Other Laws of Physics". Journal of Contemporary History 33, pp. 371-397.

— (1989). "Light and life: Niels Bohr's legacy to contemporary biology". Genome 31 (1), pp. 11-15, https://doi.org/10.1139/g89-005.

Ulam, Stanislaw M. (2002). Aventuras de un matemático. Nivola, Tres Cantos.

DOI: https://doi.org/10.15366/bp.2020.24.023

Bajo Palabra. II Época. No 24. Pgs: 449-474 
